\section{Visualization of exceptional cross- sectional en-face views of pulmonary and tricuspid valves using 2D transthoracic echocardiography in patients with pulmonary hypertension}

Cross-sectional 2D transthoracic echocardiographic (TTE) images of pulmonary and tricuspid valves depicting three leaflets simultaneously are seldom visualized in adults and are deemed to be impossible to obtain using 2D TTE. Recently, therapeutic advancements have been achieved for different pathologies of both valves. However, anatomical obstacles due to retrosternal placement and crescent shape of the right ventricle and neighboring of the left upper lung lobe to the pulmonary trunk detain comprehensive evaluation of tricuspid and pulmonary valves. Generally, short-axis en-face views cannot be obtained using 2D TTE. Lack of this morphological data requires complimentary advanced imaging techniques. However, some cardiovascular diseases yield extensive remodeling, wherein unexpected anatomical structures or infrequent projections of ordinary structures can be visualized. Pulmonary arterial hypertension (PAH) offers an opportunity to obtain these rare views, which is facilitated by dilatation of the right ventricle and pulmonary trunk. Here, we present exceptional cross-sectional en-face views of pulmonary and tricuspid valves in patients with PAH of various etiologies. Figure 1 shows en-face views of the pulmonary valve in patients with concomitant chronic thromboembolic pulmonary hypertension and residual PAH following congenital heart disease surgery (Video 1), idiopathic PAH (Video 2), and secondary PAH due to unoperated ventricular septal defect and patent ductus arteriosus, respectively. Figure 2 shows en-face views of the tricuspid valve in patients with pulmonary veno-occlusive disease, residual PAH following the closure of patent ductus arteriosus (Video 3), and secondary PAH due to unoperated ventricular and atrial septal defects, respectively.

Due to abovementioned anatomical obstacles, it is extremely difficult to obtain cross-sectional en-face images of pulmonary and tricuspid valves using 2D TTE. Unfortunately, echocardiography textbooks lack sample representative images. While 3D echocardiography has become a valuable tool to overcome this drawback, we must realize that extensive remodeling of the right side of the heart in certain diseases may facilitate $2 \mathrm{D}$ visualization of these unexpected views. Literature is also limited about this issue. En-face view of the pulmonary valve using 2D TTE has been reported just once (1). According to a recent paper attempting to obtain en-face view of the tricuspid valves,
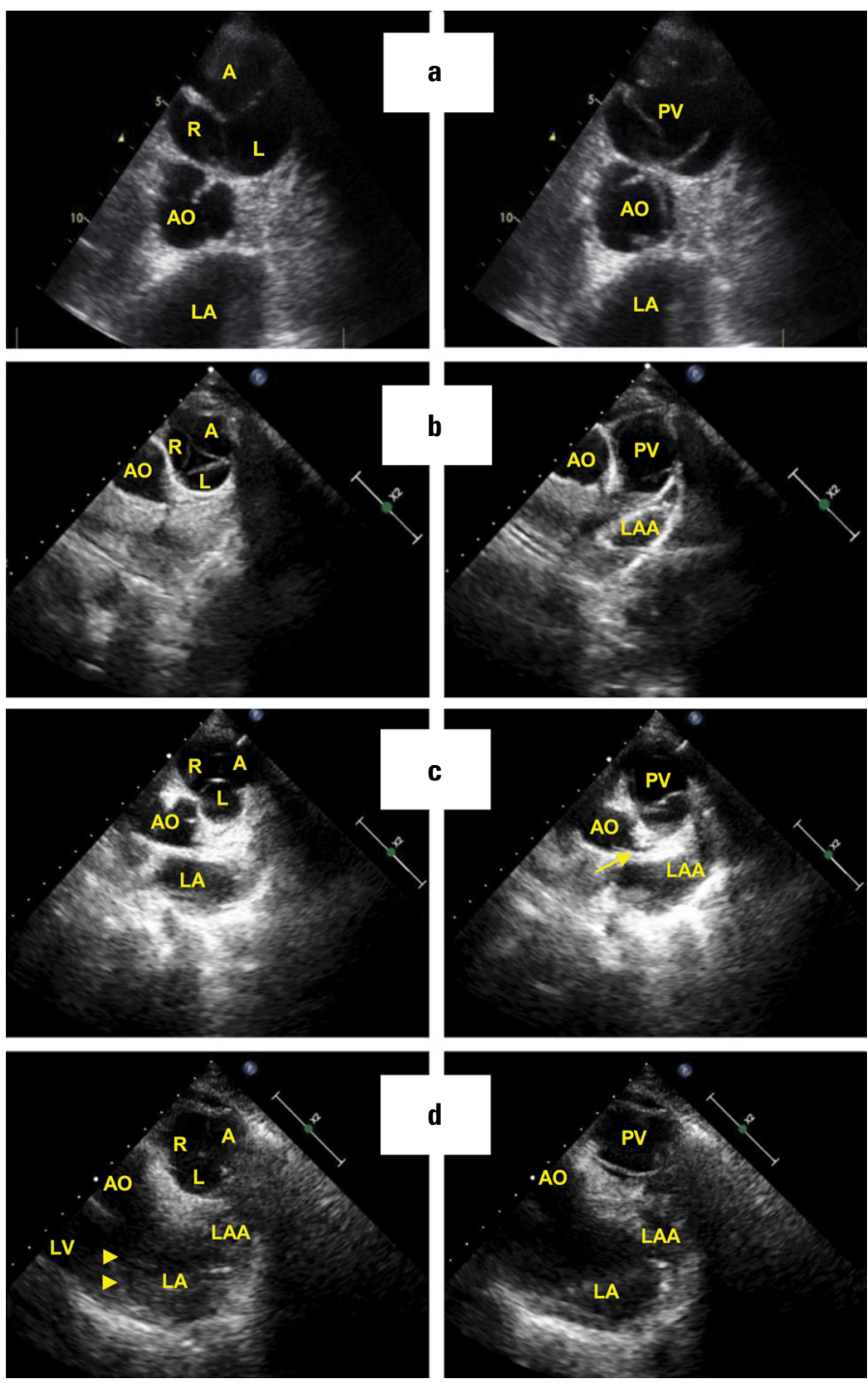

Figure 1. Modified parasternal short-axis view reveals the open (right panels) and closed (left panels) tricuspid structures of the pulmonary valve resembling the "Mercedes sign" of the aortic cusp in the closed state. Ultimate diagnoses of the patients are as follows: concomitant chronic thromboembolic pulmonary hypertension and residual PAH following congenital heart disease [details of the defect and surgery are unknown] corrective surgery (Fig. 1a; Video 1), idiopathic PAH (Fig. 1b; Video 2), and secondary PAH due to unoperated ventricular septal defect (Fig. 1c) and patent ductus arteriosus (Fig. 1d)

$A 0$ - aortic valve; $P V$ - pulmonary valve; $L A$ - left atrium; LAA - left atrial appendage; $L V$ left ventricle; $A, R, L$ - anterior, right, and left cusps of the pulmonary valve, respectively; arrow indicates the origin of the left main coronary artery, and arrow heads indicate the mitral valve

adequate images could be obtained by subcostal views in only $58 \%$ cases using 2D TTE (2). Therefore, we conclude that simply by modifying the standard parasternal projections of routine 2D TTE examination, we for the first time demonstrate that it is possible to obtain exceptional cross-sectional en-face views of pulmonary and tricuspid valves in selected patients with advanced PAH. 

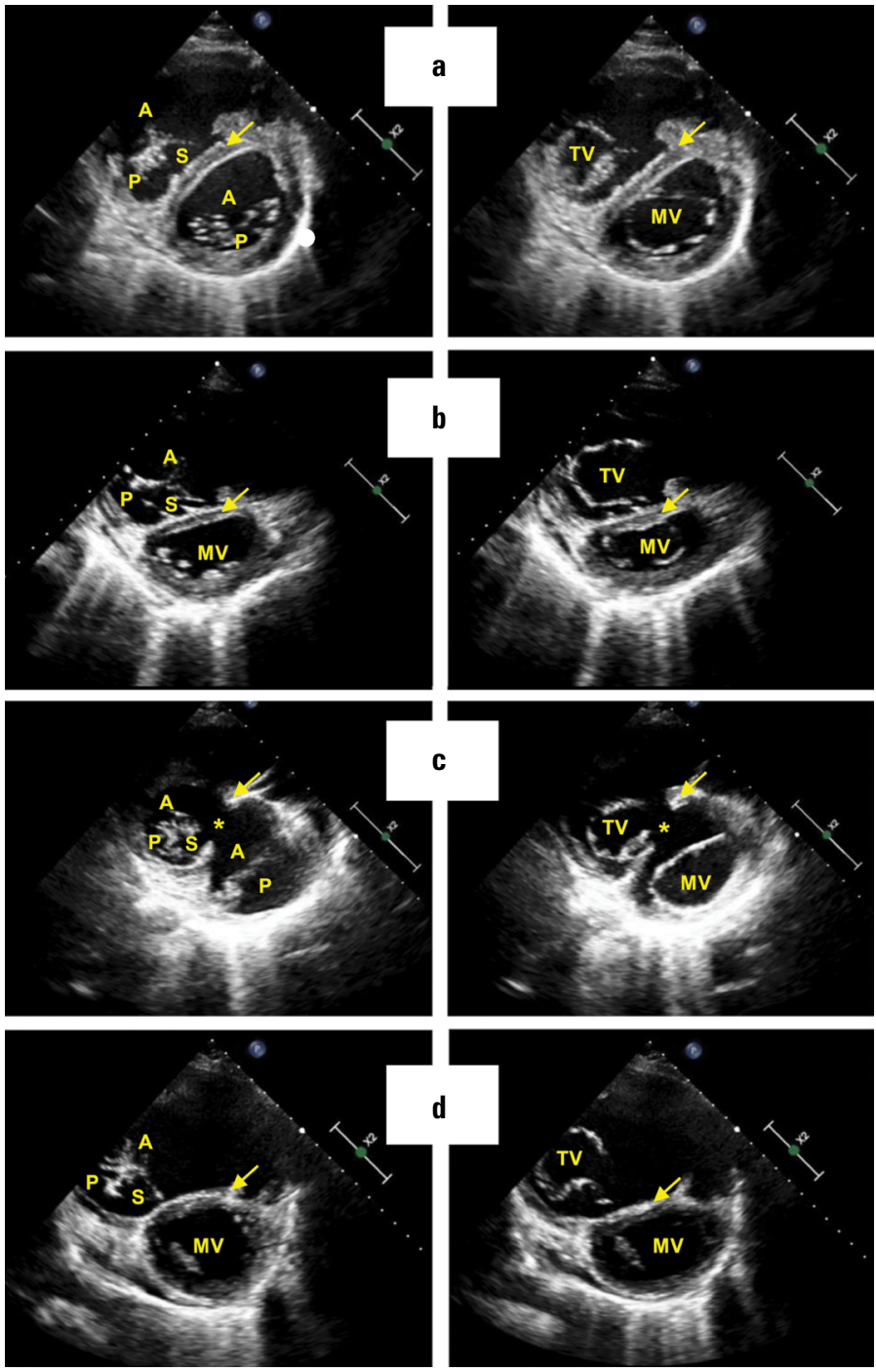

Figure 2. Modifying the standard projections by slight tilting and excursion of the transducer; along with the mitral valve, parasternal cross-sectional short-axis views (closed states in left panels, open states in right panels) of the tricuspid valve could be obtained in patients with PAH. Representative cases were diagnosed with pulmonary veno-occlusive disease (Fig. 2a), residual PAH following the closure of patent ductus arteriosus (Fig. 2b; Video 3 ), and secondary PAH due to unoperated ventricular (Fig. 2c) and atrial (Fig. 2d) septal defects, respectively

TV - tricuspid valve; MV - mitral valve; S, A, P - septal, anterior, and posterior cusps of the tricuspid valve, respectively; AP -anterior and posterior cusps of the mitral valve respectively; arrows indicate the interventricular septum, and asterisk indicates the ventricular septal defect

\section{References}

1. McAleer E, Kort S, Rosenzweig BP, Katz ES, Tunick PA, Phoon CK, et al. Unusual echocardiographic views of bicuspid and tricuspid pulmonic valves. J Am Soc Echocardiogr 2001; 14: 1036-8.

2. Stankovic I, Daraban AM, Jasaityte R, Neskovic AN, Claus P, Voigt $\mathrm{JU}$. Incremental value of the en face view of the tricuspid valve by two-dimesional and three-dimensional echocardiography for accurate identification of tricuspid valve leaflets. J Am Soc Echocardiogr 2014; 27: 376-84.
Video 1. Video displays short-axis cross-sectional view of the tricuspid structure of the pulmonary valve (simultaneous Mercedes sign along with the aortic valve is notable) of a patient who was finally diagnosed with concomitant residual $\mathrm{PAH}$ following congenital heart disease correction surgery (details of the defect are unknown) and chronic thromboembolic pulmonary hypertension (obtained by modified parasternal short-axis view)

Video 2. Video displays the tricuspid structure of the pulmonary valve in a patient diagnosed with idiopathic PAH (obtained by modified parasternal short-axis view)

Video 3. Video displays short-axis cross-sectional en-face view of the tricuspid valve (along with the bicuspid mitral valve) at parasternal short-axis in a patient with residual $\mathrm{PAH}$ following the closure of patent ductus arteriosus

\section{(D) İbrahim Başarıcı \\ Department of Cardiology, Faculty of Medicine, Akdeniz University; Antalya-Turkey}

Address for Correspondence: Dr. İbrahim Başarıcı,

Akdeniz Üniversitesi Hastanesi,

Kardiyoloji Anabilim Dalı,

07058-Konyaalti

Antalya-Türkiye

Phone: +90242 2496806

E-mail: ibasarici@gmail.com

(C) Copyright 2020 by Turkish Society of Cardiology - Available online

at www.anatoljcardiol.com

DOI:10.14744/AnatolJCardiol.2019.96165

\section{Biventricular outflow obstruction in a patient with a large sinus of the Valsalva}

\section{aneurysm}

Aneurysms of the sinus of Valsalva are defined as an abnormal enlargement of any of the three aortic sinuses. Congenital and acquired forms have been recognized. The right coronary sinus is the most common affected sinus, followed by non-coronary and left coronary sinuses (1). The prevalence of this anomaly is $0.09 \%$ in the general population (2).

Unruptured aneurysms of the sinus of Valsalva are usually asymptomatic. However, symptomatic cases have also been reported (1, 2).

We report a rare case of aneurysm of the sinus of Valsalva that resulted in right ventricular outflow tract (RVOT) obstruction with concomitant sub-valvular aortic web, causing severe left ventricular outflow tract obstruction, which we believe has not been published previously.

A 38-year-old woman underwent echocardiography in our department due to dyspnea (New York Heart Association functional class (I), which demonstrated tricuspid aortic leaflets with a very large aneurysm of the right sinus of Valsalva, causing a compressing effect on RVOT, severe sub-pulmonary stenosis, right ventricular hypertrophy, and RVOT thickening. There was also a 